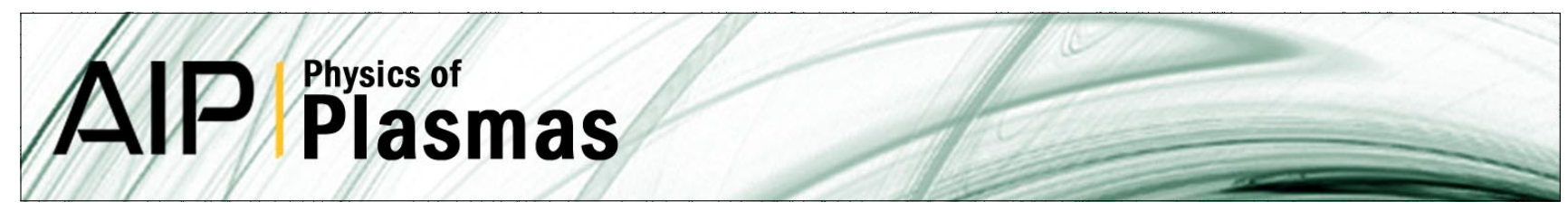

\title{
Revisited global drift fluid model for linear devices
}

Dirk Reiser

Citation: Phys. Plasmas 19, 072317 (2012); doi: 10.1063/1.4739765

View online: http://dx.doi.org/10.1063/1.4739765

View Table of Contents: http://pop.aip.org/resource/1/PHPAEN/v19/i7

Published by the American Institute of Physics.

\section{Additional information on Phys. Plasmas}

Journal Homepage: http://pop.aip.org/

Journal Information: http://pop.aip.org/about/about_the_journal

Top downloads: http://pop.aip.org/features/most_downloaded

Information for Authors: http://pop.aip.org/authors

\section{ADVERTISEMENT}

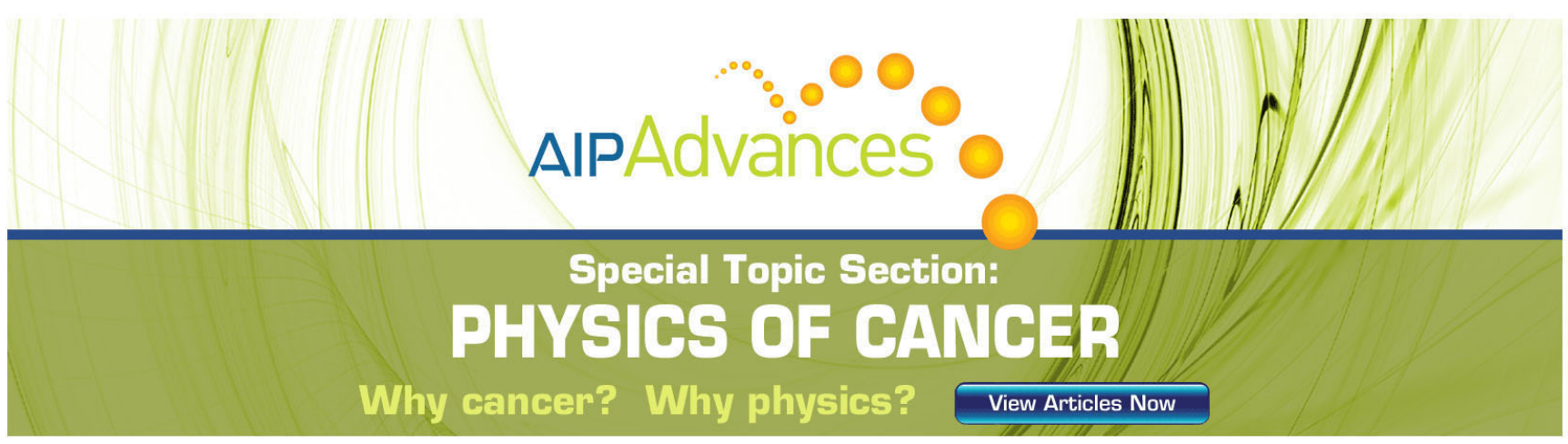




\title{
Revisited global drift fluid model for linear devices
}

\author{
Dirk Reiser \\ Institute for Energy and Climate Research—Plasma Physics, Research Center Jülich GmbH, \\ Association FZJ-Euratom, D-52425 Jülich, Germany
}

(Received 28 March 2012; accepted 5 July 2012; published online 27 July 2012)

\begin{abstract}
The problem of energy conserving global drift fluid simulations is revisited. It is found that for the case of cylindrical plasmas in a homogenous magnetic field, a straightforward reformulation is possible avoiding simplifications leading to energetic inconsistencies. The particular new feature is the rigorous treatment of the polarisation drift by a generalization of the vorticity equation. The resulting set of model equations contains previous formulations as limiting cases and is suitable for efficient numerical techniques. Examples of applications on studies of plasma blobs and its impact on plasma target interaction are presented. The numerical studies focus on the appearance of plasma blobs and intermittent transport and its consequences on the release of sputtered target materials in the plasma. Intermittent expulsion of particles in radial direction can be observed and it is found that although the neutrals released from the target show strong fluctuations in their propagation into the plasma column, the overall effect on time averaged profiles is negligible for the conditions considered. In addition, the numerical simulations are utilised to perform an a-posteriori assessment of the magnitude of energetic inconsistencies in previously used simplified models. It is found that certain popular approximations, in particular by the use of simplified vorticity equations, do not significantly affect energetics. However, popular model simplifications with respect to parallel advection are found to provide significant deterioration of the model consistency. [http://dx.doi.org/10.1063/1.4739765]
\end{abstract}

\section{INTRODUCTION}

Drift fluid models have been considered often in the last decades in a large amount of studies on plasma dynamics. Instead of solving the complete momentum equation for the perpendicular motion of the plasma particles, the perpendicular velocity is expressed as a sum of drifts. The idea that the $E \times B$-drift is dominant leads to a set of model equations for scalar quantities only. An equation for the electric field replaces the equation for the plasma velocity perpendicular to the magnetic field. The quasi-neutrality condition leads to the so-called vorticity equation, which determines the electric potential. A crucial property of those models is their particular treatment of energy conservation. It is known since years that a proper inclusion of the polarization drift is an unavoidable prerequisite for the energy conservation of the model system. Details about basic aspects of the drift fluid approximation can be found, e.g., in Refs. 1-7 and references therein.

This paper re-considers the properties of an energyconserving model for the simple geometry of a plasma in a linear device with homogeneous magnetic confinement field. It is shown that the simplicity of the cylindrical geometry leads to a compact analytical formulation of model equations suitable for standard numerical techniques. Furthermore, the resulting equations can be reduced systematically to previously used models. In Sec. II, the drift-fluid model for cylindrical geometry is re-considered. Special attention is paid to an extended form of the vorticity equation resulting from rigorous treatment of the quasineutrality condition and energy conservation properties. Section III is devoted to details on the numerical approach to solve the model equations derived. In Sec. IV, the model is studied numerically for scenarios close to realistic conditions in the PSI-2 experiment ${ }^{8,9}$ and the NAGDIS-II device. ${ }^{10,11}$ The numerical studies focus on the appearance of plasma blobs and intermittent transport and its consequences on the release of sputtered target materials in the plasma. For the cases considered, the plasma dynamics is strongly influenced by density fluctuations leading to irregular rotating structures in the entire plasma column. Additionally, an intermittent expulsion of particles in radial direction can be observed. The typical time scales of plasma rotation and intermittent particle bursts agree well with experimentally observed particle dynamics. The impact of these processes on the distribution of neutral Tungsten as a typical target material in studies on plasma surface interaction is considered. It is found that although the Tungsten neutrals also show strong fluctuations in their propagation into the plasma column, the overall effect on time averaged profiles is negligible for the conditions considered. The simulations performed are also re-examined with particular attention on the differences of the model presented and simplified approaches with respect to energy conservation. It is shown that even if certain popular approximations are lacking energetic consistency, the error in the practical examples studied here is negligible. Of course, this statement cannot be generalized to arbitrary plasma conditions, but the formalism presented might serve as a benchmark for similar model simplifications in other scenarios.

\section{RECAPITULATION OF THE DRIFT-FLUID MODEL}

In this section, basic properties of an electrostatic fluid model under drift approximation are re-considered. By 
confining the general description to a cylindrical geometry reflecting the setup of a linear plasma device, the analysis can be simplified considerably, which leads to compact forms of model equations fulfilling the requirements of energetic consistency.

\section{A. Fluid equation and drift approximation}

Starting with general fluid equations for the particle, momentum, and energy balance for each plasma species, ${ }^{12,13}$

$$
\begin{gathered}
\frac{d n}{d t}=-n \boldsymbol{\nabla} \cdot \mathbf{V}+S_{n} \\
m n \frac{d \mathbf{V}}{d t}=-\boldsymbol{\nabla} \cdot \mathbf{P}+\mathbf{R}+\operatorname{Zen}(\mathbf{E}+\mathbf{V} \times \mathbf{B})+\mathbf{S}_{m}-S_{n} m \mathbf{V} \\
\frac{3}{2} n \frac{d T}{d t}=-\boldsymbol{\nabla} \cdot \mathbf{q}-\mathbf{P}: \mathbf{\nabla} \mathbf{V}-Q+S_{E}-\mathbf{S}_{m} \cdot \mathbf{V} \\
-\frac{3}{2} S_{n} T+\frac{1}{2} m V^{2} S_{n}
\end{gathered}
$$

where $n$ denotes the density of the particular species, $\mathbf{V}$ is the flow velocity, and $T$ the particular temperature. The pressure tensor is denoted by $\mathbf{P}, m$ is the particle mass, $Z$ is the charge number, $\mathbf{q}$ is the heat flow, and $\mathbf{E}$ and $\mathbf{B}$ are the electric and magnetic field vectors, respectively. Sources for particle, momentum, and energy are denoted by $S_{n}, \mathbf{S}_{m}$, and $S_{E}$. Finally, the quantity $Q$ and the vector $\mathbf{R}$ denote the change of thermal energy and the force due to Coulomb collisions between the charged plasma particles. The total time derivative is defined by $d / d t=\partial / \partial t+\mathbf{V} \cdot \nabla$. One can write a formal solution for the perpendicular velocity $\mathbf{V}_{\perp}$ as

$$
\begin{aligned}
\mathbf{V}_{\perp}= & \frac{\mathbf{E} \times \mathbf{B}}{B^{2}}+\frac{\mathbf{B} \times \nabla p}{Z e n B^{2}}+\frac{m}{Z e B^{2}} \mathbf{B} \times \frac{d \mathbf{V}}{d t}+\frac{\mathbf{S}^{m} \times \mathbf{B}}{Z e n B^{2}} \\
& +\frac{m S^{n}}{Z e n B^{2}} \mathbf{B} \times \mathbf{V}_{\perp} .
\end{aligned}
$$

Here, the pressure tensor is approximated by $\mathbf{P}=p \mathbf{1}$ and the perpendicular part of the friction $\mathbf{R}$ has been neglected. The first term on the rhs of Eq. (4) defines the $E \times B$-velocity $\mathbf{V}_{E}$, the second the diamagnetic drift $\mathbf{V}_{*}$, and the third the polarization drift $\mathbf{V}_{p}$. Actually, Eq. (4) can be considered as an iteration scheme to find the perpendicular velocity $\mathbf{V}_{\perp}$. The iteration usually starts with the assumption that in zeroth order, $\mathbf{V}_{\perp}^{(0)}=\mathbf{V}_{E}$ and the iteration scheme is prescribed by

$$
\begin{aligned}
\mathbf{V}_{\perp}^{(i+1)}= & \mathbf{V}_{E}+\mathbf{V}_{*}+\frac{\mathbf{S}_{m} \times \mathbf{B}}{Z e n B^{2}}+\frac{m}{Z e B^{2}} \mathbf{B} \\
& \times\left(\frac{\partial}{\partial t}+\mathbf{V}^{(i+1)} \cdot \nabla+\frac{S_{n}}{n}\right) \mathbf{V}_{\perp}^{(i)},
\end{aligned}
$$

where $\mathbf{V}^{(i)}=\mathbf{V}_{\|}+\mathbf{V}_{\perp}^{(i)}$ denotes the total velocity of the $i$ th iteration step and $\mathbf{V}_{\|}$is the parallel velocity. Therefore, in first order, a relation results between the perpendicular velocity $\mathbf{V}_{\perp}^{(1)}$ and the temporal evolution of the electric field $\dot{\mathbf{E}}_{\perp}=-\nabla_{\perp} \dot{\phi}$. The unknown velocity $\mathbf{V}_{\perp}^{(1)}$ can be calculated by the requirement $\boldsymbol{\nabla} \cdot \mathbf{J}=0$, i.e., the quasineutrality condition. Finally, an equation for the temporal evolution of the electric potential $\phi$ is obtained, usually a kind of vorticity equation. Notice that in other models, the iteration equation is approximated slightly different by

$$
\begin{aligned}
\mathbf{V}_{\perp}^{(i+1)}= & \mathbf{V}_{E}+\mathbf{V}_{*}+\frac{\mathbf{S}_{m} \times \mathbf{B}}{Z e n B^{2}}+\frac{m}{Z e B^{2}} \mathbf{B} \\
& \times\left(\frac{\partial}{\partial t}+\mathbf{V}^{(i)} \cdot \nabla+\frac{S_{n}}{n}\right) \mathbf{V}_{\perp}^{(i)}
\end{aligned}
$$

This has important consequences on the details of the resulting vorticity equation and on the particular energy theorem. This is discussed in the Appendices A, B, and C.

\section{B. Drift-fluid model in cylindrical geometry}

Even in first order, the evaluation of Eq. (5) for the perpendicular drift velocity combined with the quasi-neutrality condition is in general a difficult task. The restriction to cylindrical symmetry, i.e., a homogeneous axial magnetic field, simplifies matters considerably. A linear plasma device like sketched in Fig. 1 is a reasonable realization of a cylindrical geometry where the plasma is confined by an almost homogeneous magnetic field $\mathbf{B}$ in a symmetric surrounding. This can be described appropriately by cylindrical coordinates $(r, \theta, z)$, where $z$ defines the magnetic field direction $\mathbf{B}=B \mathbf{e}_{z}$ and $r$ and $\theta$ are the polar coordinates with respect to the $z$-axis.

This simplification with respect to geometry and coordinates allows the exact treatment of several relations in the derivation of the model equations of the next sections and is, therefore, assumed in the following. In first order approximation equation, Eq. (5) for singly charged ions with mass $m_{i}$ reads

$$
\begin{aligned}
\mathbf{u}_{\perp}= & \mathbf{V}_{E}-\frac{m_{i}}{e B^{2}} \frac{\partial \nabla_{\perp} \phi}{\partial t}-\frac{m_{i}}{e B^{2}} \mathbf{u} \cdot \nabla \nabla_{\perp} \phi+\frac{\mathbf{S}_{m}^{i} \times \mathbf{B}}{e n B^{2}} \\
& -\frac{m_{i} S_{n}^{i}}{e n B^{2}} \nabla_{\perp} \phi,
\end{aligned}
$$

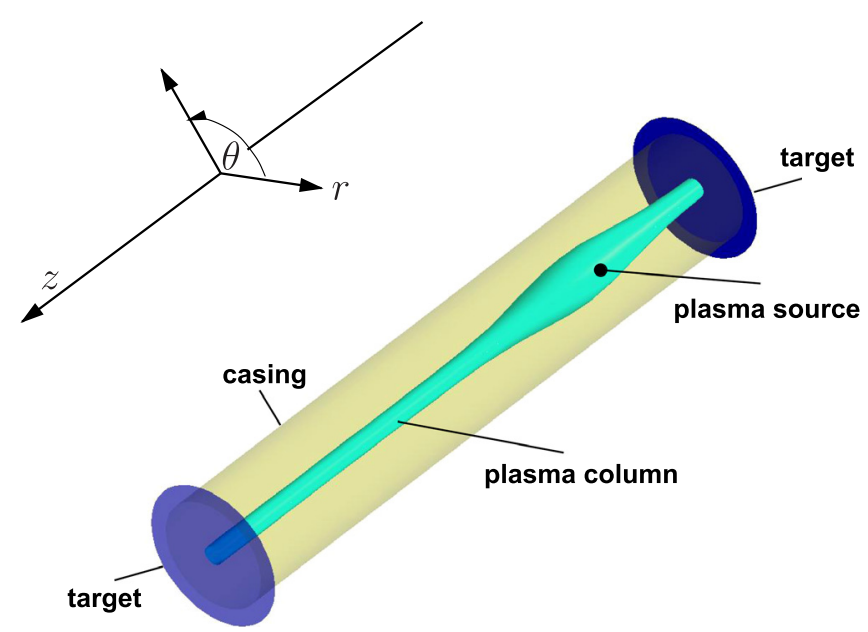

FIG. 1. Sketch of a linear plasma device according to the conditions described in Sec. III. 
where $\mathbf{u}=\mathbf{u}_{\|}+\mathbf{u}_{\perp}$ denotes the total ion velocity, with $\mathbf{u}_{\|}$ the parallel piece and $\mathbf{u}_{\perp}$ the perpendicular part. The diamagnetic drift is neglected by assuming cold ions with $T_{i} \approx 0$. An equivalent form of Eq. (7) is

$$
\begin{aligned}
\mathbf{u}_{\perp} \cdot \mathbf{D}= & \mathbf{V}_{E}-\frac{m_{i}}{e B^{2}} \frac{\partial \nabla_{\perp} \phi}{\partial t}-\frac{m_{i}}{e B^{2}} \mathbf{u}_{\|} \cdot \nabla \nabla_{\perp} \phi+\frac{\mathbf{S}_{m}^{i} \times \mathbf{B}}{e n B^{2}} \\
& -\frac{m_{i} S_{n}^{i}}{e n B^{2}} \nabla_{\perp} \phi
\end{aligned}
$$

where the tensor $\mathbf{D}$ has been introduced

$$
\mathbf{D}=\mathbf{1}+\frac{m_{i}}{e B^{2}} \nabla \nabla_{\perp} \phi
$$

which plays a central role in the subsequent analysis of the next sections. Here, the symbol 1 denotes the identity tensor. An immediate consequence of the drift approximation Eq. (8) is that the model is restricted to cases where $\mathbf{D}$ is not equal to the zero tensor. Otherwise, the equation for $\mathbf{u}_{\perp}$ would get lost and the perpendicular ion velocity is undetermined. The perpendicular velocity of the electrons $\mathbf{v}_{\perp}$ is obtained by neglecting electron inertia and is written as

$$
\mathbf{v}_{\perp}=\mathbf{V}_{E}-\frac{\mathbf{B} \times \nabla p_{e}}{e n B^{2}}-\frac{\mathbf{S}_{m}^{e} \times \mathbf{B}}{e n B^{2}}+\frac{m_{e} S_{n}^{e}}{e n B^{2}} \nabla_{\perp} \phi
$$

where $m_{e}$ denotes the electron mass and $p_{e}=n T_{e}$ the electron pressure. According to the requirement of quasineutrality, the densities of electrons and ions are assumed to be equal, i.e., $n_{e}=n_{i}=n$. Finally, this gives the following set of model equations for the temporal evolution of the density $n$, the parallel velocities of ions $u_{\|}$and electrons $v_{\|}$, and the electron temperature $T_{e}$ in the electrostatic approximation

$$
\begin{gathered}
\frac{\partial n}{\partial t}+\mathbf{v} \cdot \nabla n=-n \boldsymbol{\nabla} \cdot \mathbf{v}+S_{n}^{e}, \\
\frac{\partial v_{\|}}{\partial t}+\mathbf{v} \cdot \nabla v_{\|}=-\frac{\nabla_{\|} p_{e}}{m_{e} n}+\frac{e \eta_{\|}}{m_{e}} J_{\|}-\frac{\alpha}{m_{e}} \nabla_{\|} T_{e} \\
+\frac{e}{m_{e}} \nabla_{\|} \phi-\frac{S_{n}^{e}}{n} v_{\|}+\frac{S_{\|}^{e}}{m_{e} n}, \\
\frac{\partial u_{\|}}{\partial t}+\mathbf{u} \cdot \nabla u_{\|}=-\frac{e \eta_{\|}}{m_{i}} J_{\|}+\frac{\alpha}{m_{i}} \nabla_{\|} T_{e} \\
\frac{\partial T_{e}}{\partial t}+\mathbf{v} \cdot \nabla T_{e}=-\frac{2}{m_{i}} \nabla_{\|} \phi-\frac{S_{n}^{i}}{n} u_{\|}+\frac{S_{\|}^{i}}{m_{i} n}, \\
-\frac{2}{3} \alpha \frac{\mathbf{J}_{\|}}{e n}-\frac{2}{3} T_{e} \nabla_{\|} T_{e}+\frac{2}{3} \frac{\eta_{\|}}{n} J_{\|}^{2}-\frac{2}{3} \frac{\mathbf{S}_{m}^{e} \cdot \mathbf{v}}{n} \\
+\frac{2}{3} \frac{S_{n}^{e}}{n}\left(\frac{1}{2} m_{e} v^{2}-\frac{3}{2} T_{e}\right)+\frac{S_{E}}{n},
\end{gathered}
$$

where $\mathbf{q}_{e}$ is the electron heat flux

$$
\mathbf{q}_{e}=-\alpha T_{e} \mathbf{J}_{\|} / e-\kappa_{\|}^{e} \nabla_{\|} T_{e}-\kappa_{\perp}^{e} \nabla_{\perp} T_{e}
$$

and $\kappa_{\|}^{e}$ and $\kappa_{\perp}^{e}$ denote the classical heat conductivities. The thermal force coefficient is set to $\alpha=0.71$ (Refs. 12 and 13) and the parallel current density is defined by $\mathbf{J}_{\|}=e n\left(\mathbf{u}_{\|}\right.$ $\left.-\mathbf{v}_{\|}\right)$. As shown in the Appendix B, the Eqs. (7)-(14) obey an energy theorem in the sense that for zero sources and sink a certain energy functional is conserved in a particular volume as long as no energy is flowing through the volume boundaries. This property of the model system is particularly related to the inclusion of the complete drift velocities Eqs. (7) and (10) in the advection terms on the lhs of Eqs. (11)-(14). A result which has been found already in Refs. 4 and 5.

\section{The quasineutrality condition}

The solution of the model Eqs. (7)-(15) requires the knowledge of the time derivative of the electric field $\nabla \dot{\phi}$ appearing in Eq. (7). This is found by employing the quasineutrality condition in the form

$$
\boldsymbol{\nabla} \cdot \mathbf{J}=\boldsymbol{\nabla} \cdot\left(e n \mathbf{u}_{\perp}-e n \mathbf{v}_{\perp}\right)+\nabla_{\|} J_{\|}=0
$$

with $\mathbf{u}_{\perp}$ determined by

$$
\begin{aligned}
\mathbf{u}_{\perp}= & \left(\mathbf{V}_{E}-\frac{m_{i}}{e B^{2}} \frac{\partial \nabla_{\perp} \phi}{\partial t}-\frac{m_{i}}{e B^{2}} \mathbf{u}_{\|} \cdot \nabla \nabla_{\perp} \phi\right. \\
& \left.+\frac{\mathbf{S}_{m}^{i} \times \mathbf{B}}{e n B^{2}}-\frac{m_{i} S_{n}^{i}}{e n B^{2}} \nabla_{\perp} \phi\right) \cdot \mathbf{D}^{-1} .
\end{aligned}
$$

The tensor $\mathbf{D}$ has been defined by Eq. (9), and in cylindrical geometry, the evaluation of Eq. (17), in particular, the computation of the inverse tensor $\mathbf{D}^{-1}$, is straightforward provided that $\mathbf{D}$ is non-singular, i.e., its determinant

$$
\mathcal{D}=|\mathbf{D}|=1+\frac{m_{i}}{e B^{2}} \nabla_{\perp}^{2} \phi+\frac{m_{i}}{e B^{2}}\left|\nabla \nabla_{\perp} \phi\right|
$$

is non-zero. The perpendicular Laplacian of the electric potential $\nabla_{\perp}^{2} \phi=\nabla \cdot \nabla_{\perp} \phi=\mathbf{B} \cdot \boldsymbol{\nabla} \times \mathbf{V}_{E}$ is the vorticity of the $E \times B$-flow. The evaluation, of Eq. (16) leads to an equation for the temporal evolution of the determinant $\mathcal{D}$, which can be written in the compact form

$$
\begin{aligned}
\frac{\partial \mathcal{D}}{\partial t}+\mathbf{u} \cdot \nabla \mathcal{D}= & -\mathcal{D} \frac{m_{i}}{e B^{2}} \nabla_{\perp} \nabla_{\|} \phi \cdot \mathbf{D}^{-1} \cdot \nabla_{\perp} u_{\|} \\
& -\mathcal{D} \frac{m_{i}}{e B^{2}} \mathbf{D}^{-1}: \nabla_{\perp}\left(\frac{S_{n}^{i}}{n} \nabla_{\perp} \phi-\frac{\mathbf{S}_{m}^{i} \times \mathbf{B}}{m_{i} n}\right) \\
& +\mathcal{D} \frac{\nabla_{\|} J_{\|}}{e n}+\mathcal{D} \mathbf{u}_{\perp} \cdot \frac{\nabla n}{n}-\mathcal{D} \frac{\boldsymbol{\nabla} \cdot\left(n \mathbf{v}_{\perp}\right)}{n}
\end{aligned}
$$

Details on the derivation of this equation are given in Appendix A. It replaces the vorticity equation used in several drift fluid models. For that reason, Eq. (19) is called the "extended vorticity" equation. In addition to the temporal evolution of the vorticity $\nabla_{\perp}^{2} \phi$, this equation takes into account higher order effects due to polarization and advection by the polarization drift which must be kept to ensure energy conservation of the model system. Notice that Eq. (19) can be rearranged by using the continuity equation 


$$
\begin{aligned}
\frac{\partial \mathcal{Z}}{\partial t}+\mathbf{u} \cdot \nabla \mathcal{Z}= & \frac{m_{i}}{e B^{2}} \nabla_{\perp} \nabla_{\|} \phi \cdot \mathbf{D}^{-1} \cdot \nabla_{\perp} u_{\|} \\
& +\frac{m_{i}}{e B^{2}} \mathbf{D}^{-1}: \nabla_{\perp}\left(\frac{S_{n}^{i}}{n} \nabla_{\perp} \phi-\frac{\mathbf{S}_{m}^{i} \times \mathbf{B}}{m_{i} n}\right) \\
& -\nabla_{\|} u_{\|}+\frac{S_{n}^{e}}{n},
\end{aligned}
$$

where $\mathcal{Z}=\ln n-\ln \mathcal{D}$. This indicates that the determinant $\mathcal{D}$ is a positive quantity as well as the particle density $n$, therefore, reflecting the basic assumption of the drift fluid model that $\mathbf{V}_{E}$ is the dominant piece in the perpendicular ion velocity.

\section{NUMERICAL METHOD}

In this section, the numerical approach to solve the driftfluid equations derived above is elucidated. The details of the time stepping algorithm, in particular for the extended vorticity equation, are presented. Boundary conditions to take into account the plasma sheath at the targets are discussed and sources and sinks are specified.

\section{A. Time stepping}

Actually, Eq. (19) is an equation for the temporal evolution of the extended vorticity $\mathcal{D}$, but it can be rearranged to formulate an equation for the time derivative $\dot{\phi}$ of the form

$$
a \frac{\partial^{2} \dot{\phi}}{\partial r^{2}}+b \frac{\partial^{2} \dot{\phi}}{\partial r \partial \theta}+c \frac{\partial^{2} \dot{\phi}}{\partial \theta^{2}}+d \frac{\partial \dot{\phi}}{\partial r}+e \frac{\partial \dot{\phi}}{\partial \theta}=f .
$$

The explicit expressions for the coefficients $a, b, c, d$, $e$, and $f$ are listed in Appendix D. The Eq. (21) can be solved, e.g., by a multigrid method ${ }^{14}$ and the actual value of $\dot{\phi}(r, \theta, z, t)$ is obtained by using the actual profiles of density $n(r, \theta, z, t)$, electron temperature $T_{e}(r, \theta, z, t)$, electric potential $\phi(r, \theta, z, t)$ etc., at time $t$ in the time-stepping algorithm. Therefore, the perpendicular ion velocity $\mathbf{u}_{\perp}$ is known for any particular point in time and hence all necessary information is provided for an explicit time stepping algorithm advancing the density $n$, the parallel velocities $v_{\|}$and $u_{\|}$, and the electron temperature $T_{e}$ according to Eqs. (11)-(14). In this work, a standard Runge-Kutta scheme is used for the time integration. ${ }^{15}$ The numerically obtained intermediate values for $\dot{\phi}$ are used to advance the electric potential $\phi$ in the same manner. The profiles of all relevant quantities are prescribed on a discrete grid in $(r, \theta, z)$-coordinates, covering the domain $0 \leq r \leq R, 0 \leq \theta \leq 2 \pi$, and $0 \leq z \leq L_{\|}$. To evaluate the time derivatives $\dot{n}, \dot{u}_{\|}, \dot{v}_{\|}$, and $\dot{T}_{e}$ standard second order finite differences are employed. ${ }^{15}$ Boundary conditions are imposed by the use of ghost points.

However, the practical experience with this and similar numerical approaches up to now has shown severe instabilities coming up in long simulation runs. It was not possible to identify the detailed nature of these problems, but it is likely that most problems are related to a tendency of the model system to run into its inherent singularity at $\mathcal{D}=0$, i.e., where the polarization is blowing up and the drift fluid assumption that $\mathbf{V}_{E}$ is dominant breaks. This is accompanied also with a breakdown of the model premise that space charges are effectively shielded and its temporal evolution is slow due to Debye shielding. At the moment, these numerical and/or physical obstacles can be overcome only by the inclusion of some kind of "artificial" shielding process limiting the evolution of space charges on the small spatial and temporal scales.

\section{B. Boundary conditions}

The full description of plasma-wall interaction in a realistic plasma device is complex and, therefore, appropriate boundary conditions for fluid models are difficult to formulate. This work is confined to boundary conditions as simple as possible but reasonably close to dominant physical processes. At the casing, which is for $r=R$, the electric potential $\phi$ is set to zero, assuming a grounded perfect conductor as wall material. For the other quantities $n, v_{\|}$, $u_{\|}$, and $T_{e}$, a zero radial gradient is prescribed. For the target at $z=L_{\|}$, sheath boundary conditions are imposed. ${ }^{16,17}$ The parallel ion velocity $u_{\|}$is set to sound speed $c_{s}=\sqrt{T_{e} / m_{i}}$, and for the density $n$ and electron temperature $T_{e}$, the gradient is set to zero allowing free convective outflow of particles and heat. The parallel current density $J_{\|}=e n\left(u_{\|}-v_{\|}\right)$at the sheath entrance is determined by an electric potential arising due to charge accumulation at the target

$$
J_{\|}=e n c_{s}\left[1-\sqrt{\frac{m_{i}}{2 \pi m_{e}}} \exp \left(-\frac{e \phi-e \phi_{*}}{T_{e}}\right)\right],
$$

where $\phi_{*}$ is the electric potential at the target. This means our computational domain ends at the sheath entrance without resolving the details of the sheath on the scale of the Debye length $\lambda_{D}=\sqrt{\epsilon_{0} T_{e} /\left(n e^{2}\right)}$. For the electric potential at the sheath entrance, a zero gradient boundary condition, $\partial \phi / \partial z=0$ is imposed. For the boundary close to the plasma source, i.e., at $z=0$, analogous sheath boundary conditions are imposed. This means that an additional target is assumed at $z=0$ and the plasma source itself is modelled by a Gaussian shaped volume source for particles and energy located at $z=L_{\|} / 4$.

\section{Sources and sinks}

Up to now, the source terms in Eqs. (7)-(14) have not been specified. In the numerical applications of the next sections, the particle sources for electrons $S_{n}^{e}$ and for ions $S_{n}^{i}$ have been set equal to $S_{n}$, which is chosen as a Gaussian localized at $z=L_{\|} / 4$ and $r=0$. That is

$$
S_{n}=S_{0} \exp \left\{-\frac{4(z-L / 4)^{2}}{\Delta_{z}^{2}}-\frac{4 r^{2}}{\Delta_{r}^{2}}\right\}
$$

with $\Delta_{z}$ and $\Delta_{r}$ prescribing the extent of the Gaussian in $z$ - and $r$-direction. For the source terms acting on the parallel momentum of electrons and ions, the classical viscosities have been taken into account ${ }^{13}$

$$
S_{\|}^{e}=\eta_{0}^{e} \frac{\partial^{2} v_{\|}}{\partial z^{2}}, \quad S_{\|}^{i}=\eta_{0}^{i} \frac{\partial^{2} u_{\|}}{\partial z^{2}},
$$


where a small, but finite, ion temperature $T_{i} / T_{e} \sim 0.1$ has been used to obtain $\eta_{0}^{i}$. The momentum source $\mathbf{S}_{m}^{e}$ has been neglected in all computations. As ion momentum source $\mathbf{S}_{m}^{i}$, a viscous damping has been included

$$
\mathbf{S}_{m}^{i}=m_{i} n \mu_{\perp} \nabla \cdot \nabla_{\perp} \mathbf{V}_{E}+m_{i} n \mu_{\|} \nabla \cdot \nabla_{\|} \mathbf{V}_{E},
$$

which contributes to the perpendicular ion velocity via

$$
\frac{\mathbf{S}_{m}^{i} \times \mathbf{B}}{e n B^{2}}=\frac{m_{i}}{e B^{2}}\left(\mu_{\perp} \nabla_{\perp} \nabla_{\perp}^{2} \phi+\mu_{\|} \nabla_{\perp} \nabla_{\|}^{2} \phi\right) .
$$

The coefficients $\mu_{\perp}$ and $\mu_{\|}$have been chosen according to numerical stability requirements. This mechanism effectively smoothens the profile of the vorticity. To avoid a cascade into small scales of the parallel current density $J_{\|}$, an additional damping mechanism of the form

$$
\frac{\partial J_{\|}}{\partial t}=\mu_{\|} \nabla_{\|}^{2} J_{\|}
$$

has been implemented.

\section{APPLICATION TO PSI-2 CONDITIONS}

The apparatus presented in the sections above is used to study the plasma dynamics for physical parameters close to real experiments. It is shown that a saturated turbulent state evolves, which exhibits intermittent plasma transport in radial direction very similar to experimental observations. Also the question of importance of these intermittent processes for some aspects of plasma target interaction is addressed. In Sec. IV D a numerical assessment of possible errors in simplified models is performed to compare the re-derived model equations of this work with different approaches.

\section{A. Linear and non-linear phase}

In the following sections, the drift-fluid model and the numerical method presented above is employed for a setup close to typical discharges in the PSI-2 device. ${ }^{8,9}$ For this purpose, the following parameters are chosen: $B=0.1 \mathrm{~T}, R=10 \mathrm{~cm}, L_{\|}=2 \mathrm{~m}, \Delta_{r}=5 \mathrm{~cm}, \Delta_{z}=33 \mathrm{~cm}$, and $S_{0}=1.1 \cdot 10^{24} \mathrm{~m}^{-3} \mathrm{~s}^{-1}$. The damping parameters are chosen as $\mu_{\perp}=0.15 \mathrm{~m}^{2} / \mathrm{s}$ and $\mu_{\|}=5 \mathrm{~m}^{2} / \mathrm{s}$. The equations are solved on a $32 \times 32 \times 32$ grid in $(r, \theta, z)$ coordinates and a time step of $\Delta t=2 \cdot 10^{-10} \mathrm{~s}$ is used. The simulations are started with constant density, temperature and electric potential, and linearly varying profiles in $z$-direction for the electron and ion velocities to match the sound speed at the target boundaries. Additionally, a small amplitude noise in the density is superimposed, introducing non-axisymmetric perturbations into the plasma dynamics. The plasma column will be filled in the course of time due to the presence of the particle source and the small amplitude perturbations grow according to linear instabilities. After a certain time, the linear device is filled with plasma and the non-linear interaction between the different modes present saturates.

Fig. 2 shows two snapshots of the non-axisymmetric piece $\tilde{n}$ of the plasma density during the evolution to a nonlinearly saturated state. It can be seen that for small amplitudes in the non-axisymmetric density fluctuations, i.e., in the phase of linear dynamics, the plasma density exhibits a highly symmetric distribution with low poloidal mode numbers (here, $m=4$, left figure). With increasing amplitude of nonaxisymmetric perturbations, the non-linear interaction takes over the dynamics and the density pattern becomes irregular (right figure). The particular dominant and most unstable mode in the linear phase is strongly depending on details of the particle source in the simulation. For different source strength and radial extension, very different patterns are possible, an outcome already observed in Ref. 7. In Fig. 3, the time traces of the different contributions to the total energies of the model system are shown. (For details on the energy diagnostic, see Appendix B). The non-linear saturation, leading to a statistically stationary state with energies fluctuating around a constant mean value, is obvious. The dominant contributions to the total energy can be identified as the sum of pressure $3 p_{e} / 2$ and ion parallel kinetic energy $m_{i} n u_{\|}^{2} / 2$.

\section{B. Bursty transport of plasma filaments}

The saturated plasma state is not quiet but is characterized by irregular patterns in each poloidal cross section rotating according to the drive by $E \times B$ velocity. Another feature appearing in the simulation results is an intermittent expulsion of particles in radial direction. This is illustrated by the series of snapshots in Fig. 4, showing the plasma density at

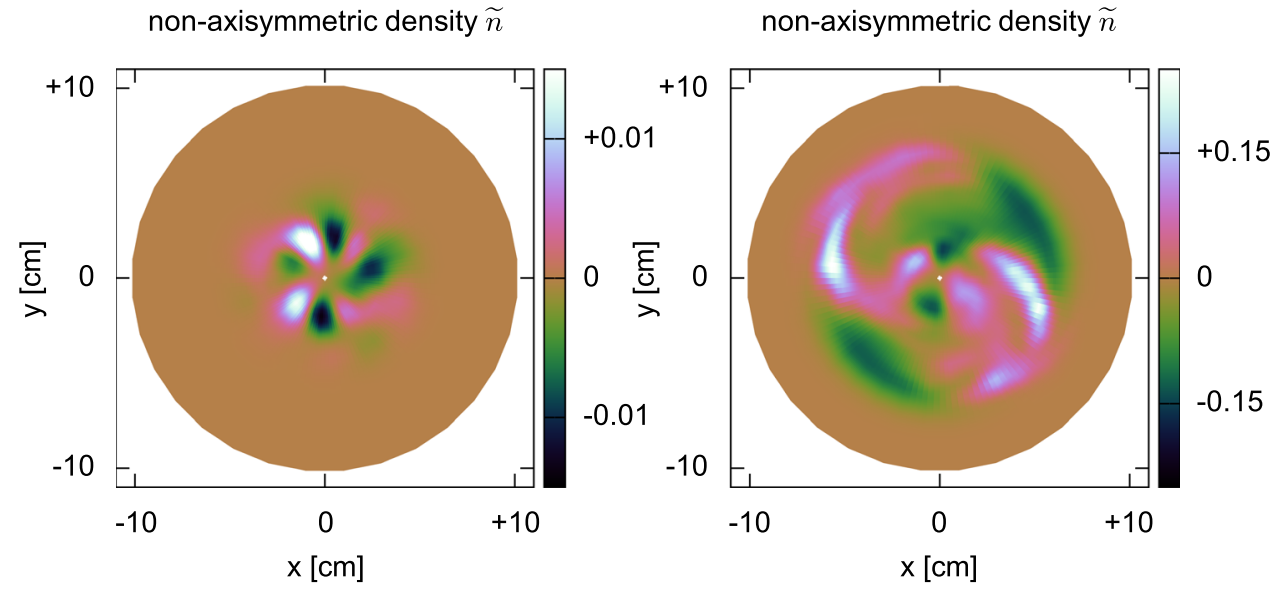

FIG. 2. Snapshots of the nonaxisymmetric piece of the plasma density at $z=L_{\|} / 4$ in the linear phase (left) and the non-linear phase (right) at $t=312 \mu \mathrm{s}$ and $t=832 \mu$ s simulation time, respectively. The density is given in units of $n_{0}=1 \cdot 10^{19} \mathrm{~m}^{-3}$. 


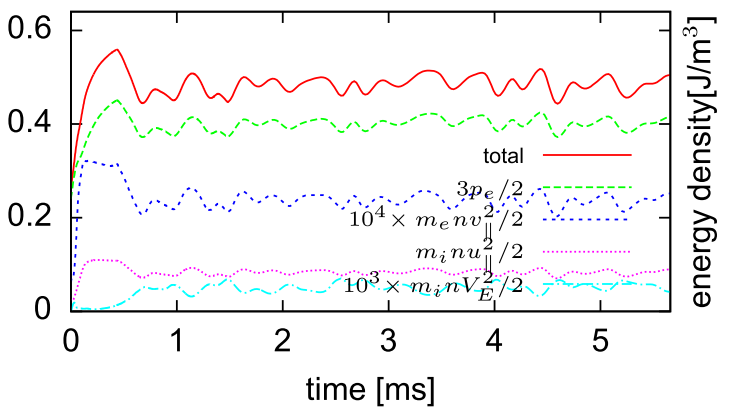

FIG. 3. Time traces of energy densities at the target at $r=R / 4$ and $\theta=\pi / 2$.

the target over a period of $160 \mu \mathrm{s}$. The figures clearly show the appearance of plasma blobs expelled from the plasma column (see the snapshot for $t=100 \mu \mathrm{s}$, a blob located at south-west). The appearance and disappearance of these plasma blobs take place on a time scale of order $100 \mu \mathrm{s}$. Similar results have been found experimentally, e.g., Refs. 10 and 11. A more detailed investigation of these bursty events demonstrates that the blobs are actually elongated filaments peeling away from the plasma column.

This is illustrated by Fig. 5 showing in addition to the snapshots of plasma density at $z=0$ (upper left figure) and $z=L_{\|}$(upper right figure), the cut in the $r-z$-plane (lower figure) going through the blob appearing at $z=L_{\|}$. It can be seen that the blobby transport is essentially a process occurring over the entire plasma column. In these simulations, this process usually starts in the region of the plasma source and some kind of breathing pushes away the elongated filament. In the $r-z$-plot of Fig. 5, also this process can be observed. The next filament around $r=3 \mathrm{~cm}$ will be pulled off soon. This inherent process of rotating plasma structures,

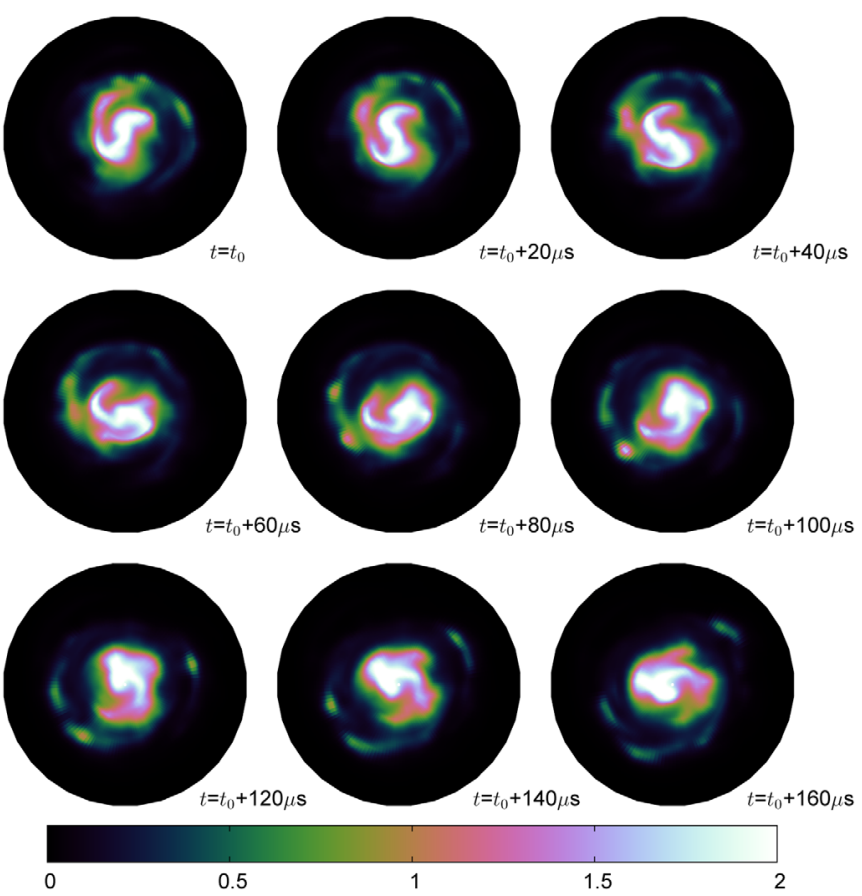

FIG. 4. Snapshots of plasma density at the target for successive time points. The density is given in units of $n_{0}=1 \cdot 10^{19} \mathrm{~m}^{-3}$.
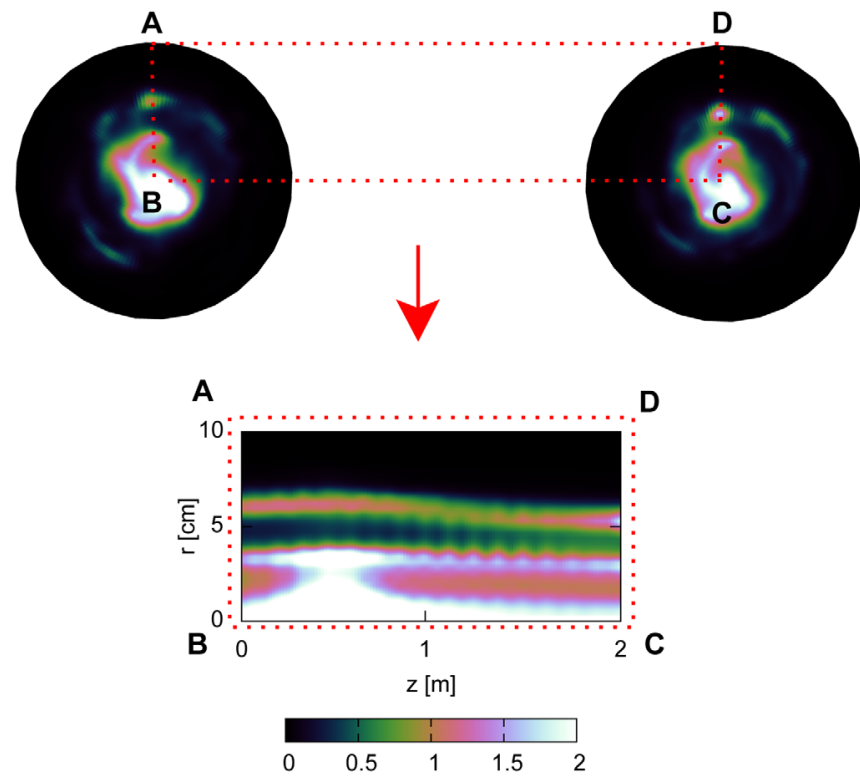

FIG. 5. Snapshots of plasma density at the targets at $z=0$ (upper left) and at $z=L_{\|}$(upper right). The contour plot below covers the region between the two cuts marked in red. The plasma blobs located on these cuts are connected along the $z$-axis. Notice that the snapshot upper right corresponds to the snapshot for $t_{0}+100 \mu \mathrm{s}$ of Fig. 4 but has been rotated for better visualization. The density is given in units of $n_{0}=1 \cdot 10^{19} \mathrm{~m}^{-3}$.

non-linearly stirred by $E \times B$ motion is reflected by the time traces of density fluctuations at the targets, shown for a particular point at the target in Fig. 6. This proves that the plasma rotation sweeps the plasma structures over the target on a time scale of several $\mathrm{kHz}$.

\section{Impact of plasma fluctuations on penetration of sputtered impurities}

The plasma dynamics observed in the numerical simulations presented in the last sections raises an important question for studies on plasma wall interactions: what might be the impact of such fluctuations on the release and the spatial distribution of sputtered target materials and how important is a high temporal resolution in experimental diagnostics to take into account these effects into interpretation properly? Among the large number of issues related to this question in this work, only the aspect of penetration of neutral target atoms is discussed. For this purpose, we employ a simple model to estimate the distribution of Tungsten as an important target

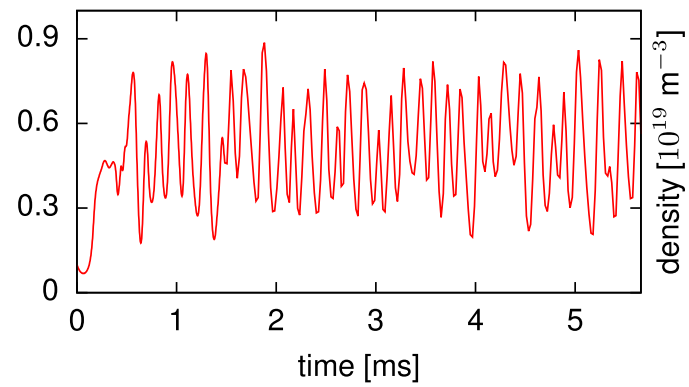

FIG. 6. Time traces of total density fluctuations at the target at $r=R / 4$ and $\theta=\pi / 2$. 
material. It is assumed that the distribution of neutral Tungsten sputtered from the target at $z=L_{\|}$is governed by the equation

$$
\frac{\partial n_{\mathrm{w}}}{\partial t}=v \frac{\partial n_{\mathrm{w}}}{\partial z}-k n_{\mathrm{w}}
$$

where $n_{\mathrm{w}}=n_{\mathrm{w}}(r, \theta, z, t)$ denotes the neutral density and $k$ $=k(r, \theta, z, t)=n_{e}\langle\sigma v\rangle$ is the ionization rate of atomic Tungsten, depending on the electron density $n_{e}=n_{e}(r, \theta, z, t)$ and the rate coefficient $\langle\sigma v\rangle$ taken from Ref. 18 . The velocity $v$ of the neutral Tungsten leaving the target at $z=L_{\|}$is assumed to be constant and positive, i.e., directed into the plasma column, and for the boundary condition at the target, it is assumed that the Tungsten influx is given by $\Gamma_{\mathrm{w}}(r, \theta, t)$ $=\alpha n_{e}\left(r, \theta, L_{\|}, t\right) v$ with a constant sputter rate $\alpha$. Then, the density of Tungsten is

$$
\begin{aligned}
n_{\mathrm{w}}(r, \theta, z, t)= & \alpha n_{e}\left(r, \theta, L_{\|}, t+z / v-L_{\|} / v\right) \\
& \times \exp \left\{-\frac{1}{v} \int_{z}^{L_{\|}} k(y, t+z / v-y / v) d y\right\} .
\end{aligned}
$$

It is of interest to what extent this Tungsten distribution which depends on the details of the spatial and temporal evolution of the background electron density $n_{e}$ can be approximated by profiles using time averaged values for the plasma background parameters. More precisely, the question is whether it is necessary to take into account the fast dynamics of the plasma oscillations to obtain an adequate overall picture of the impurity distribution or not. If not, it is enough to know details of time averaged plasma parameters only. This distinction is an issue for the interpretation of experiments if high temporal resolution diagnostics is not available. Actually, the snapshots of Tungsten neutrals in a particular $r-z$-plane for different times in the simulation shown in Fig. 7 prove that the distribution of the target released impurities is not smooth and exhibits considerable fluctuations.

To continue the discussion, we compare the time averaged Tungsten density $\bar{n}_{\mathrm{w}}$ with the approximate density $\hat{n}_{\mathrm{w}}$ obtained by the use of time averaged background parameters

$$
\hat{n}_{\mathrm{w}}(r, \theta, z)=\alpha \bar{n}_{e}\left(r, \theta, L_{\|}\right) \exp \left\{-\frac{1}{v} \int_{z}^{L_{\|}} \bar{k}(r, \theta, y) d y\right\} .
$$

Here, the bar denotes the time average over an interval $T$. For the following results, the transit time of the neutral particles has been chosen as averaging interval, i.e., $T=L_{\|} / v$. The expression Eq. (30) reflects a practical estimate of the impurity distribution from the evaluation of atomic processes using time averaged plasma profiles.

The results for Tungsten neutrals leaving the target with an energy of $8.6 \mathrm{eV}$ are shown in Fig. 8. For these scenarios-characterized in particular by relatively small temperature fluctuations-and the spatial and temporal scales involved almost no difference can be observed between the time averaged result of the detailed dynamics and the approximative result. However, one should be careful and this special case should not be generalized without further checks. For more complicated processes (e.g., related to thresholds)
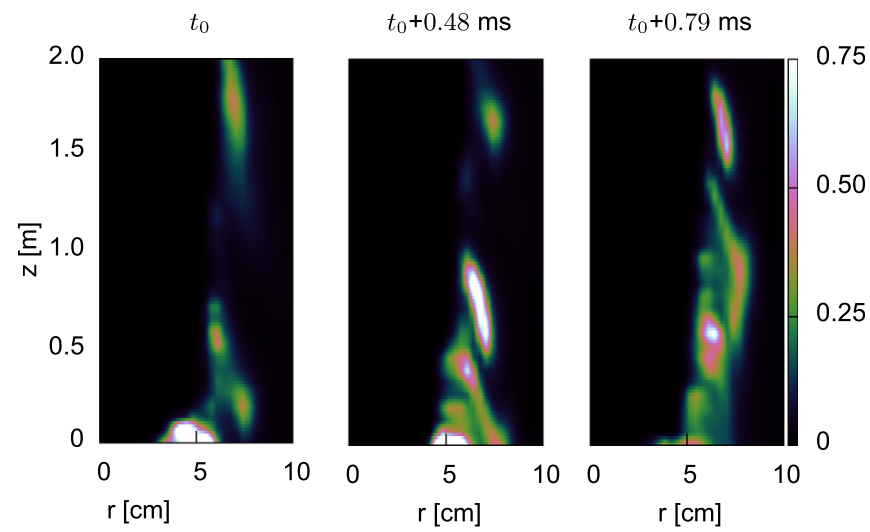

FIG. 7. Snapshots of neutral Tungsten distributions at different times. The color coding refers to density given in units of $10^{17} \mathrm{~m}^{-3}$ corresponding to a sputter rate of $\alpha=0.052$.

and different time scales, the difference can be significant. A more detailed discussion of this topic based on statistical methods can be found in Ref. 19. The process discussed here corresponds to the fast recycling case of Ref. 19 with relatively small changes in the neutral distribution in the region of a few mean free path lengths away from the target.

\section{Vorticity equations and energy conservation- assessment of traditional models}

A particular feature of the drift-fluid model presented including the extended vorticity equation presented here is that it obeys an energy theorem. This property is elucidated in Appendix B, and the deficiencies due to certain approximations often used-in particular in the vorticity equationare discussed on an analytical basis in Appendix C. In this section, the simulation runs presented in the last sections are re-examined from the point of view of energy conservation in approximative models. A few widely used simplifications are considered and analytical expressions for the inherent error of certain approaches (e.g., Eqs. (C7) and (C8) of Appendix C) are estimated. For this purpose, an a-posteriori check is performed using the complete model in the simulations to assess the magnitude of errors occurring in simplified models. Three different simplifications are considered in this analysis
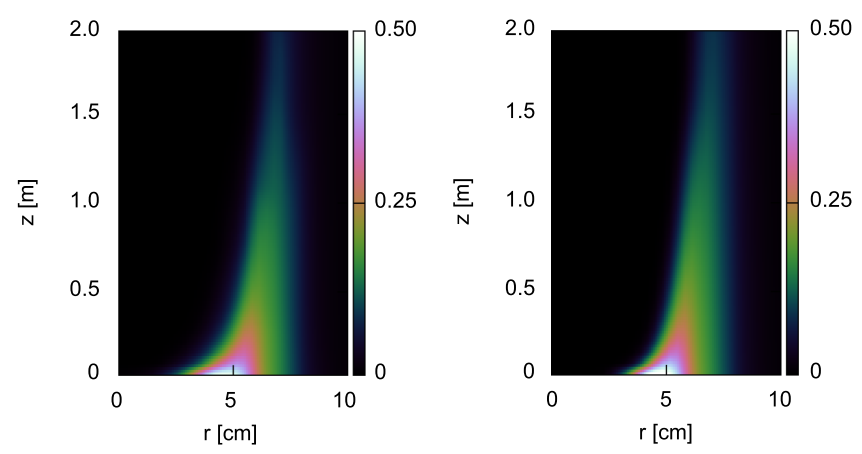

FIG. 8. Comparison of time averaged Tungsten densities obtained from the detailed dynamics $\left(\bar{n}_{\mathrm{w}}\right.$, left) and the approximation obtained by averaged plasma parameters $\left(\hat{n}_{\mathrm{w}}\right.$, right). The color coding refers to density given in units of $10^{17} \mathrm{~m}^{-3}$ corresponding to a sputter rate of $\alpha=0.052$. 
(1) treatment of the vorticity dynamics represented by the simplified Eqs. (C1) and (C2),

(2) treatment of the vorticity dynamics by the use of Eqs. (C1) and (C2) and additionally neglecting the parallel advection in there, i.e., $\mathbf{u}_{\|} \rightarrow 0$

(3) treatment of the vorticity dynamics by Eqs. (C1) and (C2), neglecting parallel advection in there, and additionally neglecting the parallel advection in the parallel momentum equations Eqs. (12) and (13).

The cases 1 and 2 have been discussed already in Appendix $\mathrm{C}$, and the error $\tilde{L}$ in the energy theorem is given for case 1 by Eq. (C7) and for case 2 by Eq. (C8). As can be seen by inspection of Eq. (B7), the error of case 3 is

$$
\tilde{L}=\left(1-\frac{m_{e}}{m_{i}}\right) \frac{m_{i} V_{E}^{2}}{2} \nabla_{\|}\left(n v_{\|}\right)-\frac{m_{i} u_{\|}^{2}}{2} \nabla_{\|}\left(n u_{\|}\right) \text {. }
$$

The time traces of these different error terms possibly contributing to a bias in the energetic properties of the model system are compared to the total temporal changes of the different pieces of energy in the system. For this purpose, the following rates of change of energy are introduced

$$
\begin{gathered}
\tilde{L}_{1}=\int_{V} \frac{m_{i} V_{E}^{2}}{2} \nabla_{\|}\left(n v_{\|}\right) d V, \\
\tilde{L}_{2}=\int_{V} \frac{m_{i} u_{\|}^{2}}{2} \nabla_{\|}\left(n u_{\|}\right) d V, \\
\tilde{L}_{3}=\int_{V} \frac{m_{i} V_{E}^{2}}{2 e} \nabla_{\|} J_{\|} d V,
\end{gathered}
$$

and

$$
\begin{aligned}
& E_{1}=\int_{V} \frac{\partial}{\partial t}\left(\frac{m_{i} n V_{E}^{2}}{2}\right) d V, \\
& E_{2}=\int_{V} \frac{\partial}{\partial t}\left(\frac{m_{i} n u_{\|}^{2}}{2}\right) d V .
\end{aligned}
$$

The time traces of these quantities for the statistically stationary state of the simulation runs discussed in the last sections are shown in Fig. 9. It can be seen that the time derivatives $E_{1}$ and $E_{2}$ exhibit fluctuations around a mean value. The frequency of these fluctuations is of order $10^{-8} \mathrm{~s}^{-1}$. The time traces of the error estimate $\tilde{L}_{2}$ are on a level one order of magnitude above the time trace of $E_{1}$, whereas the levels of $\tilde{L}_{1}$ and $\tilde{L}_{3}$ are much lower than $E_{1}$ and $E_{2}$. This result is illustrated additionally in the bottom figure of Fig. 9, where the time averages of the rates of change of energy and the error estimates are shown. It can be concluded that the errors due to the simplifications in cases 1 and 2, i.e., when employing the approximative vorticity equation, are of marginal importance only. At least in the cases considered here, the particular pieces missing in the energy balance are very small compared to other effects,
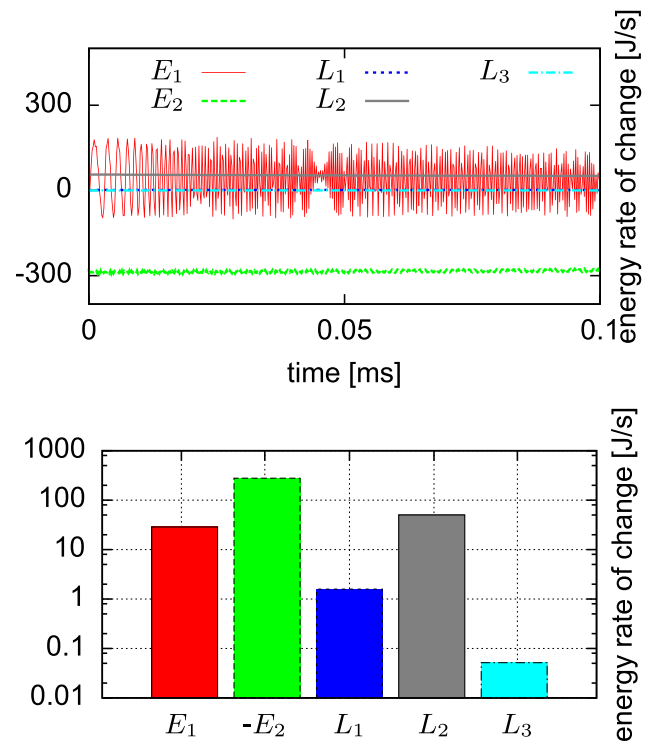

FIG. 9. Top: Time trace of the energy rates of change. Bottom: Logarithmic plot of the time averaged values corresponding to the time traces of the top figure.

e.g., dissipation. The only significant level of errors can be observed in case 3 , i.e., if $\tilde{L}_{2}$ comes into play. This error is of similar magnitude as $E_{1}$ and it can be concluded that the energy theorem is affected significantly because $\tilde{L}_{2}$ contributes strongly in the temporal evolution of energy. However-without showing detailed plots here-it must be pointed out that this has strong implications for the energy flow in the system only, but the results for the overall plasma dynamics and temporal behaviour of the plasma parameters are only marginally affected. It can be concluded that the energy conserving model presented and, in particular, the extended vorticity equation do not show significant changes compared to known simplified model approaches lacking certain energetic properties. Of course, it must be emphasized that this result cannot be generalized to any possible condition in a linear experiment. Therefore, one might take advantage of the analytical framework presented here for an estimation of possibly significant errors in other simplified models and other parameter ranges.

\section{SUMMARY}

An electrostatic drift-fluid model for cylindrical plasma geometry with cold ions has been re-derived. Due to the axi-symmetric geometry in a linear device, a new set of analytically compact model equations are obtained taking care of energetic consistency in a rigorous manner. Moreover, these model equations include approximative model equations used in previous studies as limiting cases. Therefore, traditional models can be assessed in a systematic way to estimate possible errors due to energetic inconsistencies. The model presented has been solved numerically by standard techniques to study the plasma dynamics in realistic scenarios. The simulations were guided by machine parameters of the PSI- 2 experiment. A pronounced turbulence has been observed governed by fluctuations in all plasma parameters being of almost the same order of magnitude as 
the spatial and temporal averages. A bursty plasma transport in radial direction appears on time scales in close agreement with experimental observations. Drawing on the example of Tungsten release from the target, the consequences of such strong fluctuations on plasma wall interactions at the target have been considered. It is found that for the particular parameters considered the time averaged picture of impurity neutrals released from the target is almost identical to an estimate based on averaged plasma profiles. However, a generalization of this special result is not possible and future studies have to be calibrated with detailed investigations of the turbulent scales, which might differ in other scenarios than considered in this work. Besides this, the simulation runs have been investigated from the viewpoint of energetic consistency of the new model equations compared to traditional models and its consequences for practical applications. Analytical expressions for the systematic error in simplified models have been derived and their magnitude has been estimated numerically. It is found that at least for the particular cases considered, the changes in energetics and plasma dynamics are only marginal, i.e., certain approximations of reduced models are proven to be tolerable with respect to the overall outcome. But, this result cannot be generalized and it is recommended to repeat the analysis presented here for particular applications with different setup.

\section{APPENDIX A: EXTENDED VORTICITY EQUATION}

In a tedious but straightforward calculation, it can be shown that Eq. (19) results from Eqs. (16) and (17) by repeated use of the following relation valid for any vector $\mathbf{w}_{\perp}$ perpendicular to $\mathbf{B}$

$$
\boldsymbol{\nabla} \cdot\left(\mathcal{D} \mathbf{w}_{\perp} \cdot \mathbf{D}^{-1}\right)=\mathcal{D} \mathbf{D}^{-1}: \nabla_{\perp} \mathbf{w}_{\perp} .
$$

By insertion, it can be proved that this relation is exact for the cylinder geometry considered here. A special case of this relation is

$$
\begin{aligned}
\nabla \cdot\left(\mathcal{D} \frac{m_{i}}{e B^{2}} \nabla_{\perp} f \cdot \mathbf{D}^{-1}\right)= & \mathcal{A}(f)+\mathcal{C}(f)+\mathcal{A}(f) \mathcal{C}(\phi) \\
& +\mathcal{A}(\phi) \mathcal{C}(f)-2 \mathcal{B}(\phi) \mathcal{B}(f)
\end{aligned}
$$

where the operators $\mathcal{A}, \mathcal{B}$, and $\mathcal{C}$ are defined by

$$
\begin{gathered}
\mathcal{A}(f)=\frac{m_{i}}{e B^{2}} \frac{\partial^{2} f}{\partial r^{2}} \\
\mathcal{B}(f)=\frac{m_{i}}{e B^{2}}\left(\frac{1}{r} \frac{\partial^{2} f}{\partial \theta \partial r}-\frac{1}{r^{2}} \frac{\partial f}{\partial \theta}\right) \\
\mathcal{C}(f)=\frac{m_{i}}{e B^{2}}\left(\frac{1}{r} \frac{\partial f}{\partial r}+\frac{1}{r^{2}} \frac{\partial^{2} f}{\partial \theta^{2}}\right)
\end{gathered}
$$

Using this relation for first derivatives of $\phi$, i.e., $f=\partial \phi / \partial t, f=\partial \phi / \partial z$, etc., the rhs of Eq. (A2) provides terms like $\partial \mathcal{D} / \partial t, \partial \mathcal{D} / \partial z$, etc., appearing in Eq. (19). This is due to the relation

$$
\mathcal{D}=1+\mathcal{A}(\phi)+\mathcal{C}(\phi)+\mathcal{A}(\phi) \mathcal{C}(\phi)-\mathcal{B}(\phi)^{2}
$$

Notice that

$$
\frac{m_{i}}{e B^{2}} \nabla_{\perp}^{2} \phi=\mathcal{A}(\phi)+\mathcal{C}(\phi)
$$

Therefore, the extended vorticity equation Eq. (19) contains higher order corrections in spatial derivatives of the electric potential $\phi$, which is the additional pieces of the polarization tensor $\nabla \nabla \phi$ usually neglected in vorticity equations of previously used models. Notice also that by inserting $\mathbf{w}_{\perp}=\mathbf{h}_{\perp}$ in Eq. (A1) with

$$
\mathbf{h}_{\perp}=\nabla_{\perp}\left(\frac{r^{2}}{4}+\frac{m_{i}}{e B^{2}} \frac{\phi}{2}\right)=\frac{1}{2}\left(r \mathbf{e}_{r}+\frac{m_{i}}{e B^{2}} \nabla_{\perp} \phi\right),
$$

one finds that the determinant $\mathcal{D}$ can be represented as a divergence, i.e., $\mathcal{D}=\nabla \cdot \mathbf{H}_{\perp}$, where $\mathbf{H}_{\perp}=\mathcal{D} \mathbf{h}_{\perp} \cdot \mathbf{D}^{-1}$.

\section{APPENDIX B: ENERGY THEOREM}

To gain some insight into the energetics of the model system defined by Eqs. (11)-(14) and Eq. (19), an analysis very similar to the investigations in Ref. 5 is used. Neglecting the source terms in Eqs. (11)-(14), the following relation can be derived

$$
\begin{aligned}
\frac{\partial}{\partial t}( & \left.\frac{m_{e} n v_{\|}^{2}}{2}+\frac{m_{i} n u_{\|}^{2}}{2}+\frac{5}{2} p_{e}\right) \\
+ & \nabla \cdot\left(\frac{m_{e} n v_{\|}^{2}}{2} \mathbf{v}^{\prime \prime}+\frac{m_{i} n u_{\|}^{2}}{2} \mathbf{u}^{\prime}+\frac{3}{2} p_{e} \mathbf{v}^{\prime \prime \prime}\right) \\
= & -v_{\|} \nabla_{\|} p_{e}-J_{\|} \nabla_{\|} \phi-\nabla \cdot \mathbf{q}_{e}+\mathbf{v}^{\prime \prime \prime} \cdot \nabla p_{e} \\
& -\frac{m_{e} v_{\|}^{2}}{2} \nabla \cdot\left(n \mathbf{v}^{\prime}-n \mathbf{v}^{\prime \prime}\right)-\frac{m_{i} u_{\|}^{2}}{2} \nabla \cdot\left(n \mathbf{v}^{\prime}-n \mathbf{u}^{\prime}\right) \\
& -\frac{3}{2} T_{e} \boldsymbol{\nabla} \cdot\left(n \mathbf{v}^{\prime}-n \mathbf{v}^{\prime \prime \prime}\right) .
\end{aligned}
$$

For clarity purposes, the advection velocities of the Eqs. (11)-(14) have been marked by primes to illustrate in a transparent way the basic results of Ref. 5 that the advective terms in all drift fluid model equations must fulfill certain requirements to guarantee energy conservation. The replacements $\mathbf{v} \rightarrow \mathbf{v}^{\prime}$ in Eq. (11), $\mathbf{v} \rightarrow \mathbf{v}^{\prime \prime}$ in Eq. (12), $\mathbf{u} \rightarrow \mathbf{u}^{\prime}$ in Eq. (13), and $\mathbf{v} \rightarrow \mathbf{v}^{\prime \prime \prime}$ in Eq. (14) have been introduced to identify the particle velocities of the different equations. To proceed, the term $J_{\|} \nabla_{\|} \phi$ is replaced using the relation

$$
\begin{aligned}
\mathbf{J} \cdot \nabla \phi & =\nabla \cdot(\phi \mathbf{J})-\phi \boldsymbol{\nabla} \cdot \mathbf{J} \\
& =e n \mathbf{u}_{\perp} \cdot \nabla \phi-e n \mathbf{v}_{\perp} \cdot \nabla \phi+J_{\|} \nabla_{\|} \phi .
\end{aligned}
$$

Assuming that the quasineutrality condition Eq. (16) is fulfilled, one obtains by using the perpendicular electron velocity $\mathbf{v}_{\perp}$ of Eq. (10) and the perpendicular ion velocity $\mathbf{u}_{\perp}$ from Eq. (7) 


$$
\begin{aligned}
J_{\|} \boldsymbol{\nabla}_{\|} \phi= & \nabla \cdot(\phi \mathbf{J})-e \phi \boldsymbol{\nabla} \cdot(n \mathbf{u}-n \mathbf{v})+\mathbf{V}_{E} \cdot \nabla p_{e} \\
& +\frac{m_{i} n}{2} \frac{\partial V_{E}^{2}}{\partial t}+\frac{m_{i} n}{2} \mathbf{u} \cdot \nabla V_{E}^{2} .
\end{aligned}
$$

Inserting this into the balance equation above and using again the continuity equation Eq. (11) gives the energy theorem for the temporal evolution of the energy density

$$
U=\frac{m_{e} n v_{\|}^{2}}{2}+\frac{m_{i} n u_{\|}^{2}}{2}+\frac{3}{2} p_{e}+\frac{m_{i} n V_{E}^{2}}{2},
$$

which reads

$$
\frac{\partial U}{\partial t}+\nabla \cdot \mathbf{F}=L
$$

The flux of energy $\mathbf{F}$ is given as

$$
\mathbf{F}=\frac{m_{i} n V_{E}^{2}}{2} \mathbf{u}+\frac{m_{i} n u_{\|}^{2}}{2} \mathbf{u}^{\prime}+\frac{m_{e} n v_{\|}^{2}}{2} \mathbf{v}^{\prime \prime}+\frac{5}{2} p_{e} \mathbf{v}^{\prime \prime \prime}+\mathbf{q}_{e}+\phi \mathbf{J} .
$$

The rhs of the energy theorem Eq. (B5) contains sources and sinks and is

$$
\begin{aligned}
L= & e \phi \boldsymbol{\nabla} \cdot(n \mathbf{u}-n \mathbf{v})-\left(\mathbf{v}-\mathbf{v}^{\prime \prime \prime}\right) \cdot \boldsymbol{\nabla} p_{e} \\
& -\frac{m_{i} V_{E}^{2}}{2} \boldsymbol{\nabla} \cdot\left(n \mathbf{v}^{\prime}-n \mathbf{u}\right)-\frac{m_{i} u_{\|}^{2}}{2} \boldsymbol{\nabla} \cdot\left(n \mathbf{v}^{\prime}-n \mathbf{u}^{\prime}\right) \\
& -\frac{m_{e} v_{\|}^{2}}{2} \nabla \cdot\left(n \mathbf{v}^{\prime}-n \mathbf{v}^{\prime \prime}\right)-\frac{3}{2} T_{e} \boldsymbol{\nabla} \cdot\left(n \mathbf{v}^{\prime}-n \mathbf{v}^{\prime \prime \prime}\right) .
\end{aligned}
$$

A desirable feature of a reasonable model is $L=0$, representing energy conservation in any finite volume if no physical sources and sinks are present. Inspection of Eq. (B7) shows that this is fulfilled only if $\mathbf{v}=\mathbf{v}^{\prime}=\mathbf{v}^{\prime \prime}=\mathbf{v}^{\prime \prime \prime}$ and $\boldsymbol{\nabla} \cdot(n \mathbf{v}-n \mathbf{u})=0$ and $\boldsymbol{\nabla} \cdot\left(n \mathbf{v}-n \mathbf{u}^{\prime}\right)=0$ simultaneously. This implies that the ion velocities $\mathbf{u}^{\prime}$ in Eq. (13) and $\mathbf{u}$ in Eq. (19) cannot be chosen arbitrarily. To achieve energy conservation in the model equations, they must fulfill the quasineutrality condition. This means that the complete expression Eq. (17) must be used including polarization drift. Finally, one can conclude that the model defined by Eqs. (11)-(14) and Eq. (19) represent an energy conserving model.

\section{APPENDIX C: TRADITIONAL APPROXIMATIONS OF THE VORTICITY EQUATION}

It is instructive to see how a more familiar form of the vorticity equation compares to the extended vorticity equation derived in the last sections. Several models used in previous drift-fluid studies start from the approximation introduced in Eq. (6) and obtain instead of Eq. (7) the first order expression

$$
\begin{aligned}
\mathbf{u}_{\perp}= & \mathbf{V}_{E}-\frac{m_{i}}{e B^{2}} \frac{\partial \nabla_{\perp} \phi}{\partial t}-\frac{m_{i}}{e B^{2}} \mathbf{u}_{\|} \cdot \nabla \nabla_{\perp} \phi-\frac{m_{i}}{e B^{2}} \mathbf{V}_{E} \cdot \nabla \nabla_{\perp} \phi \\
& +\frac{\mathbf{S}_{m}^{i} \times \mathbf{B}}{e n B^{2}}-\frac{m_{i} S_{n}^{i}}{e n B^{2}} \nabla_{\perp} \phi .
\end{aligned}
$$

This leads to the following form of a vorticity equation

$$
\begin{aligned}
\frac{m_{i}}{e B^{2}} & \frac{\partial \nabla_{\perp}^{2} \phi}{\partial t}+\frac{m_{i}}{e B^{2}}\left(\mathbf{u}_{\|}+\mathbf{V}_{E}\right) \cdot \nabla \nabla_{\perp}^{2} \phi \\
= & \frac{\nabla_{\|} J_{\|}}{e n}-\frac{m_{i}}{e B^{2}} \nabla_{\perp} \nabla_{\|} \phi \cdot \nabla_{\perp} u_{\|}-\frac{m_{i}}{e B^{2}} \frac{S_{n}^{i}}{n} \nabla_{\perp}^{2} \phi \\
& -\frac{m_{i}}{e B^{2}} \nabla_{\perp} \phi \cdot \nabla\left(\frac{S_{n}^{i}}{n}\right)+\frac{m_{i}}{e B^{2}} \mu_{\perp} \nabla_{\perp}^{2} \nabla_{\perp}^{2} \phi \\
& +\frac{m_{i}}{e B^{2}} \mu_{\|} \nabla_{\|}^{2} \nabla_{\perp}^{2} \phi+\mathbf{u}_{\perp} \cdot \frac{\nabla n}{n}-\frac{\nabla \cdot\left(n \mathbf{v}_{\perp}\right)}{n} .
\end{aligned}
$$

Instead of the extended vorticity Eq. (19), this vorticity equation-or similar versions-has been used in previous models. In the following, it is shown that this approximation results in an inconsistency with respect to energy conservation. To proceed, Eq. (C1) is used in the exercise of Appendix $\mathrm{B}$ to derive the energy theorem and one finds a modified balance equation

$$
\frac{\partial \tilde{U}}{\partial t}+\nabla \cdot \tilde{\mathbf{F}}=\tilde{L},
$$

where the energy density $\tilde{U}$ is identical to the extended model

$$
\tilde{U}=\frac{m_{e} n v_{\|}^{2}}{2}+\frac{m_{i} n u_{\|}^{2}}{2}+\frac{3}{2} p_{e}+\frac{m_{i} n V_{E}^{2}}{2}
$$

but the flux of energy $\tilde{\mathbf{F}}$ is now given as

$$
\begin{aligned}
\tilde{\mathbf{F}}= & \frac{m_{i} n V_{E}^{2}}{2}\left(\mathbf{u}_{\|}+\mathbf{V}_{E}\right)+\frac{m_{i} n u_{\|}^{2}}{2} \mathbf{u}^{\prime}+\frac{m_{e} n v_{\|}^{2}}{2} \mathbf{v}^{\prime \prime} \\
& +\frac{5}{2} p_{e} \mathbf{v}^{\prime \prime \prime}+\mathbf{q}_{e}+\phi \mathbf{J}
\end{aligned}
$$

and the rhs of the energy theorem contains additional sources and sinks and is

$$
\tilde{L}=L-\frac{m_{i} V_{E}^{2}}{2} \nabla \cdot\left(n \mathbf{u}_{\|}+n \mathbf{V}_{E}-n \mathbf{v}\right) .
$$

Inserting $\mathbf{v}_{\perp}$ from Eq. (10) (without source contributions), it is obvious that even in the case that $L=0$ (if $\mathbf{v}=$ $\mathbf{v}^{\prime}=\mathbf{v}^{\prime \prime}=\mathbf{v}^{\prime \prime \prime}$ and $\mathbf{u}$ and $\mathbf{u}^{\prime}$ fulfil the quasineutrality condition), a non-zero rest remains, i.e.,

$$
\tilde{L}=-\frac{m_{i} V_{E}^{2}}{2 e} \nabla_{\|} J_{\|}
$$

If in addition $\mathbf{u}_{\|} \rightarrow 0$ in Eq. (C1), the vorticity Eq. (C2) would not contain a parallel advection of vorticity anymore (an approximation used, e.g., in Ref. 7). Then-as long as the parallel electron velocity is kept in the particle balance Eq. (11) - the remaining energetic inconsistency would be

$$
\tilde{L}=\frac{m_{i} V_{E}^{2}}{2} \nabla_{\|}\left(n v_{\|}\right)
$$

Removing now also the parallel advection in the particle balance, i.e., $\mathbf{v}^{\prime} \rightarrow 0$, would force this defect to zero. But on 
the other hand, this would break the consistency represented by the source term Eq. (B7) completely and several sources and sinks would appear in the model system. The conclusion is that approximations based on vorticity equations like Eq. (C2) instead of Eq. (19) and/or simplified advection terms instead of the full parallel and perpendicular pieces as in Eqs. (11)-(14) fail to conserve energy properly.

\section{APPENDIX D: COEFFICIENTS OF EQ. (21)}

The coefficients $a, b, c, d, e$, and $f$ of Eq. (21) are obtained by evaluating the extended vorticity Eq. (19) for the case of cylindrical geometry considered here. For the sake of completeness, the detailed expressions are listed in the following:

$$
\begin{gathered}
a=\frac{m_{i}}{e B^{2}}[1+\mathcal{C}(\phi)] \\
b=-\frac{m_{i}}{e B^{2}} \frac{2 \mathcal{B}(\phi)}{r} \\
c=\frac{m_{i}}{e B^{2}} \frac{1+\mathcal{A}(\phi)}{r^{2}} \\
d=\frac{m_{i}}{e B^{2}} \frac{1+\mathcal{A}(\phi)}{r}+\frac{m_{i}}{e B^{2}} T^{r}(\mathcal{Z}) \\
e=\frac{m_{i}}{e B^{2}} \frac{2 \mathcal{B}(\phi)}{r^{2}}+\frac{m_{i}}{e B^{2}} T^{\theta}(\mathcal{Z}) \\
\left(\frac{\partial \phi}{\partial r} \frac{\partial n}{\partial \theta}-\frac{\partial \phi}{\partial \theta} \frac{\partial n}{\partial r}\right)+\mathcal{D} \frac{\nabla_{\|} J_{\|}}{e n}+\mathbf{K}_{\perp} \cdot \mathbf{T}(\mathcal{Z}) \\
-\frac{m_{i}}{e n B^{2}} \nabla_{\perp} \phi \cdot \mathbf{T}\left(S_{n}^{i}\right)+\frac{m_{i}}{e n B^{2}} \frac{S_{n}^{i}}{n} \nabla_{\perp} \phi \cdot \mathbf{T}(n) \\
-\frac{m_{i}}{e B^{2}} \nabla_{\perp} \nabla_{\|} \phi \cdot \mathbf{T}\left(u_{\|}\right)-\frac{S_{n}^{i}}{n}[\mathcal{A}(\phi)+\mathcal{C}(\phi) \\
\left.+2 \mathcal{A}(\phi) \mathcal{C}(\phi)-2 \mathcal{B}(\phi)^{2}\right]+\mu_{\perp}\left[\mathcal{A}\left(\nabla_{\perp}^{2} \phi\right)+\mathcal{C}\left(\nabla_{\perp}^{2} \phi\right)\right. \\
\left.+\mathcal{A}\left(\nabla_{\perp}^{2} \phi\right) \mathcal{C}(\phi)+\mathcal{A}(\phi) \mathcal{C}\left(\nabla_{\perp}^{2} \phi\right)-2 \mathcal{B}(\phi) \mathcal{B}\left(\nabla_{\perp}^{2} \phi\right)\right] \\
+\mu_{\|}\left[\mathcal{A}\left(\nabla_{\|}^{2} \phi\right)+\mathcal{C}\left(\nabla_{\|}^{2} \phi\right)+\mathcal{A}\left(\nabla_{\|}^{2} \phi\right) \mathcal{C}(\phi)\right. \\
\left.+\mathcal{A}(\phi) \mathcal{C}\left(\nabla_{\|}^{2} \phi\right)-2 \mathcal{B}(\phi) \mathcal{B}\left(\nabla_{\|}^{2} \phi\right)\right]
\end{gathered}
$$

where $\mathcal{Z}=\ln (n / \mathcal{D})$ and

$$
\begin{aligned}
\mathbf{K}_{\perp}= & \mathbf{V}_{E}-\frac{m_{i}}{e B^{2}} u_{\|} \nabla_{\perp} \nabla_{\|} \phi+\frac{m_{i}}{e B^{2}} \mu_{\perp} \nabla_{\perp} \nabla_{\perp}^{2} \phi \\
& +\frac{m_{i}}{e B^{2}} \mu_{\|} \nabla_{\perp} \nabla_{\|}^{2} \phi-\frac{m_{i} S_{n}^{i}}{e n B^{2}} \nabla_{\perp} \phi
\end{aligned}
$$

and

$$
\begin{aligned}
\mathbf{T}(g)= & \mathcal{D} \mathbf{D}^{-1} \cdot \nabla_{\perp} g=[1+\mathcal{C}(\phi)] \frac{\partial g}{\partial r} \mathbf{e}_{r}-\frac{\mathcal{B}(\phi)}{r} \frac{\partial g}{\partial \theta} \mathbf{e}_{r} \\
& -\frac{\mathcal{B}(\phi)}{r} \frac{\partial g}{\partial r} \mathbf{e}_{\theta}+\frac{1+\mathcal{A}(\phi)}{r^{2}} \frac{\partial g}{\partial \theta} \mathbf{e}_{\theta} .
\end{aligned}
$$

The operators $\mathcal{A}, \mathcal{B}$, and $\mathcal{C}$ are defined in Appendix $\mathrm{A}$ by Eqs. (A3)-(A5).

${ }^{1}$ F. L. Hinton and C. W. Horton, Jr., Phys. Fluids 14, 116 (1971).

${ }^{2}$ M. Wakatani and A. Hasegawa, Phys. Fluids 27, 611 (1984).

${ }^{3}$ B. D. Scott, Phys. Fluids B 4, 2468 (1992).

${ }^{4}$ A. Zeiler, J. F. Drake, and B. Rogers, Phys. Plasmas 4, 2134 (1997).

${ }^{5}$ B. Scott, Phys. Plasmas 10, 963 (2003).

${ }^{6}$ O. E. Garcia, V. Naulin, A. H. Nielsen, and J. Juul Rasmussen, Phys. Plasmas 12, 062309 (2005)

${ }^{7}$ V. Naulin, T. Windisch, and O. Grulke, Phys. Plasmas 15, 012307 (2008).

${ }^{8}$ B. Unterberg, R. Jaspers, R. Koch, V. Massaut, J. Rapp, D. Reiter,

S. Kraus, A. Kreter, V. Philipps, H. Reimer, U. Samm, L. Scheibl,

B. Schweer, J. Schuurmans, I. Uytdenhouwen, R. Al, M. A. van den Berg, S. Brons, H. J. N. van Eck, W. J. Goedheer, M. F. Graswinckel, T. van der Grift, A. Kleyn, W. R. Koppers, O. Kruyt, A. Lof, H. J. van der Meiden, W. Melissen, M. van de Pol, G. J. van Rooij, P. Smeets, J. Scholten, D. C. Schrame, G. De Temmerman, W. Vijvers, P. A. Zeijlmans van Emmichoven, and J. J. Zielinski, Fusion Eng. Des. 86, 1797 (2011).

${ }^{9}$ A. Kreter, Fusion Sci. Technol. 59(1T), 51 (2011).

${ }^{10}$ H. Tanaka, N. Ohno, Y. Tsuji, and S. Kajita, Contrib. Plasma Phys. 50(3-5), 256-266 (2010).

${ }^{11}$ H. Tanaka, N. Ohno, Y. Tsuji, K. Okazaki, and S. Kajita, "Statistical analysis of the spatial behavior of plasma blobs around the plasma column in a linear plasma device," Contrib. Plasma Phys. 52(5-6), 424-428 (2012).

${ }^{12}$ S. I. Braginskii, Rev. Plasma Phys. 1, 205 (1965).

${ }^{13}$ K. Miyamoto, Plasma Physics for Nuclear Fusion, (MIT, Cambridge 1980), pp. 128-133.

${ }^{14}$ W. L. Briggs, A Multigrid Tutorial (SIAM, 1987).

${ }^{15}$ G. A. Korn, and T. M. Korn, Mathematical Handbook for Scientists and Engineers (McGraw-Hill, New York, 1961), p. 182.

${ }^{16}$ K. U. Riemann, J. Phys. D: Appl. Phys. 24, 493 (1991).

${ }^{17}$ R. H. Cohen and D. D. Ryutov, Phys. Plasmas 2, 2011 (1995).

${ }^{18}$ T. Pütterich, R. Neu, R. Dux, A. D. Whiteford, M. G. O'Mullane, and ASDEX Upgrade Team, Plasma Phys. Controlled Fusion 50, 085016 (2008).

${ }^{19}$ Y. Marandet, A. Mekkaoui, D. Reiter, P. Börner, P. Genesio, F. Catoire, J. Rosato, H. Capes, L. Godbert-Mouret, M. Koubiti, and R. Stamm, Nucl. Fusion 51, 083035 (2011). 